The Astrophysical Journal, 163:L25-L31, 1971 January 1

(C) 1971. The University of Chicago. All rights reserved. Printed in U.S.A.

\title{
MAFFEI 1: A NEW MASSIVE MEMBER OF THE LOCAL GROUP?
}

\author{
Hyron Spinrad, * W. L. W. Sargent, $\dagger$ J. B. Oke, $\dagger$ Gerry Neugebauer, $\dagger$ \\ Robert Landau, ${ }^{*}$ Ivan R. King, ${ }^{*}$ James E. Gunn, $\dagger$ \\ Gordon Garmire, $\ddagger$ and Nannielou H. Dieter $\S$ \\ Received 1970 November 2
}

\section{ABSTRACT}

An extensive collection of observational data is presented which indicates that the recently discovered infrared object Maffei 1 is a highly reddened giant elliptical galaxy at a distance of about 1 $\mathrm{Mpc}$, and thus is probably a new massive member of the Local Group.

\section{INTRODUCTION}

In 1968, Maffei (1968) discovered two infrared objects in the neighborhood of IC 1805. The objects are conspicuous as extended, elliptical sources on I-N plates, but are just barely visible on the blue 48-inch Palomar Sky Survey plates. They are located in the plane of the Galaxy at approximately $l^{\mathrm{II}} \approx 136^{\circ}, b^{\mathrm{II}} \approx-0.5^{\circ}$; the two objects are separated by about $40^{\prime}$. At $2.2 \mu$, where differences due to interstellar extinction are small, the flux from the central $8^{\prime \prime}$ of Maffei 1 is comparable to that from a similar area of M31. Nonthermal radio emission at 3, 4, and $9 \mathrm{~cm}$ has been detected from Maffei 2 by Bell, Seaquist, and Brown (1970), although no radio emisssion was observed from Maffei 1.

The possibility that these objects are reddened external galaxies, and possibly even members of the Local Group of galaxies, has led to an observational program among observers at the Leuschner, Lick, and Hale Observatories which utilizes a variety of techniques covering wavelengths from 0.4 to $3.5 \mu$.

The instrumentation used in this program is conventional and too varied to be described in detail. Direct photographs of Maffei 1 were obtained by using the Leuschner Observatory 30 -inch f/8 reflector and I-N plates (I. K., H. S., R. L.); further infrared plates were also obtained with the 48 -inch Schmidt camera and 200-inch direct image tube (J. G.). Spectra were obtained with both the Cassegrain image tube (W. S., J. G.) and the multichannel spectrometer (J. O.) (Oke 1969) of the Hale 200-inch telescope, as well as the prime-focus single-channel spectrometer at the 120-inch telescope of the Lick Observatory with an S-20 detector (H. S.) (Wampler 1968). Infrared photometry was obtained at the Hale 200-inch telescope, as well as the Mount Wilson 100-inch, 60-inch, and 24-inch telescopes of the Hale Observatories (G. G., G. N.); the photometer and photometric system have been described by Becklin and Neugebauer (1968) and Hyland et al. (1969). Finaily, radio emission from the $21-\mathrm{cm}$ line of $\mathrm{H}$ I was searched for with the 85-foot telescope at Hat Creek (N. D.).

The results of this combined effort are presented in this paper; they pertain almost exclusively to Maffei 1 , the brighter of the two objects. The now-accumulating observations of Maffei 2 will be discussed in a later paper.

* Astronomy Department, University of California, Berkeley.

$\dagger$ Hale Observatories, California Institute of Technology, Carnegie Institution of Washington.

$\ddagger$ California Institute of Technology.

$\S$ Radio Astronomy Laboratory, University of California, Berkeley. 


\section{OBSERVATIONS}

\section{a) Photographic Observations}

Figure 1 (Plate L2) shows an I-N plate of Maffei 1; Figure 2 (Plate L3), a deeper infrared image-tube plate. The object, which can be traced to radii of about 70", appears to be elliptical with its major axis almost east-west.

On the Schmidt plates, isophotes which maintain the elliptical character can be traced to at least $3^{\prime}$. As discussed below, at $8000 \AA$ there is still approximately 3 mag of extinction; thus the angular radius of Maffei 1 is certainly much larger than $3^{\prime}$.

The core of the object is photometrically resolved on the Leuschner I-N plates (scale $34^{\prime \prime} \mathrm{mm}^{-1}$ ). The plate had no direct calibration, but an indirect calibration was obtained from iris photometry and microphotometer tracings of stars; a fit of the core of the profile to the standard interpolation formula (King, Byrnes, and Minkowski 1967)

$$
I=I_{0}\left[1+\left(\theta / \theta_{c}\right)^{2}\right]^{-1}
$$

gave a core radius $\theta_{c}$ of $5 " .5$.

\section{b) Infrared Photometry}

The infrared photometry of Maffei 1 is at wavelengths where the interstellar extinction is much less than in the visible or in the photographic infrared. In Figure 3, the total $2.2-\mu$ flux within apertures of various sizes is presented as well as comparable data from M31. In all cases the apertures were centered on the position which gave the maximum flux with the smallest apertures used; this position corresponds to the position of maximum intensity as seen on the 48 -inch Sky Survey blue plate. Almost none of the data were obtained under ideal observing conditions; therefore, any single measurement is subject to a large systematic uncertainty. In addition, the photometer optically
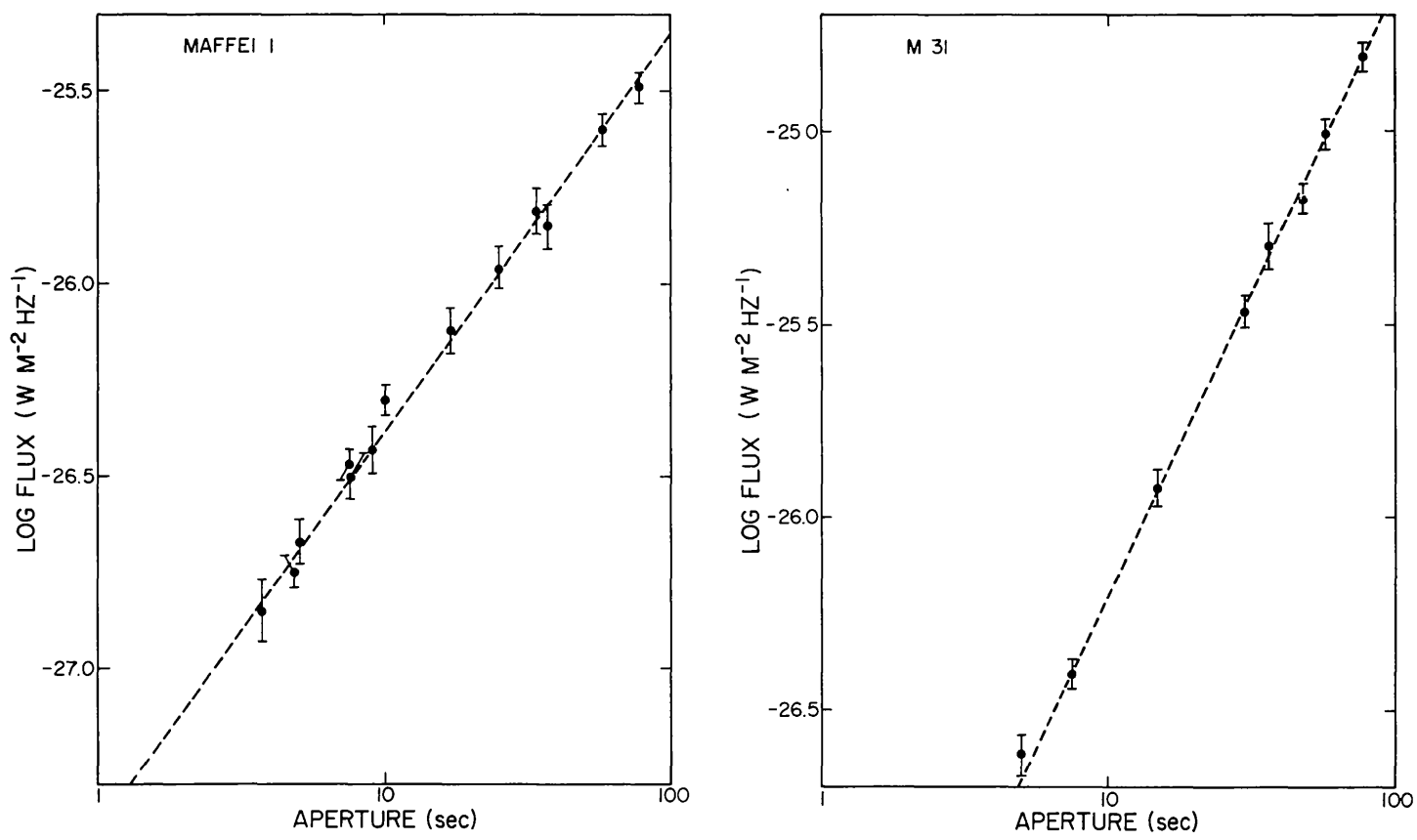

FIG. 3.-Observed flux from Maffei 1 and M31 at $2.2 \mu$ plotted against focal-plane diaphragm diameter. The reddening correction for Maffei 1 is about 0.2 in the logarithm. Note that from the central regions $\left(D \leq 10^{\prime \prime}\right)$ the fluxes are comparable. Dashed lines correspond to a surface brightness which is proportional to $D^{-1.0}$ for Maffei 1 and $D^{-0.4}$ for M31. 


\section{PLATE L2}

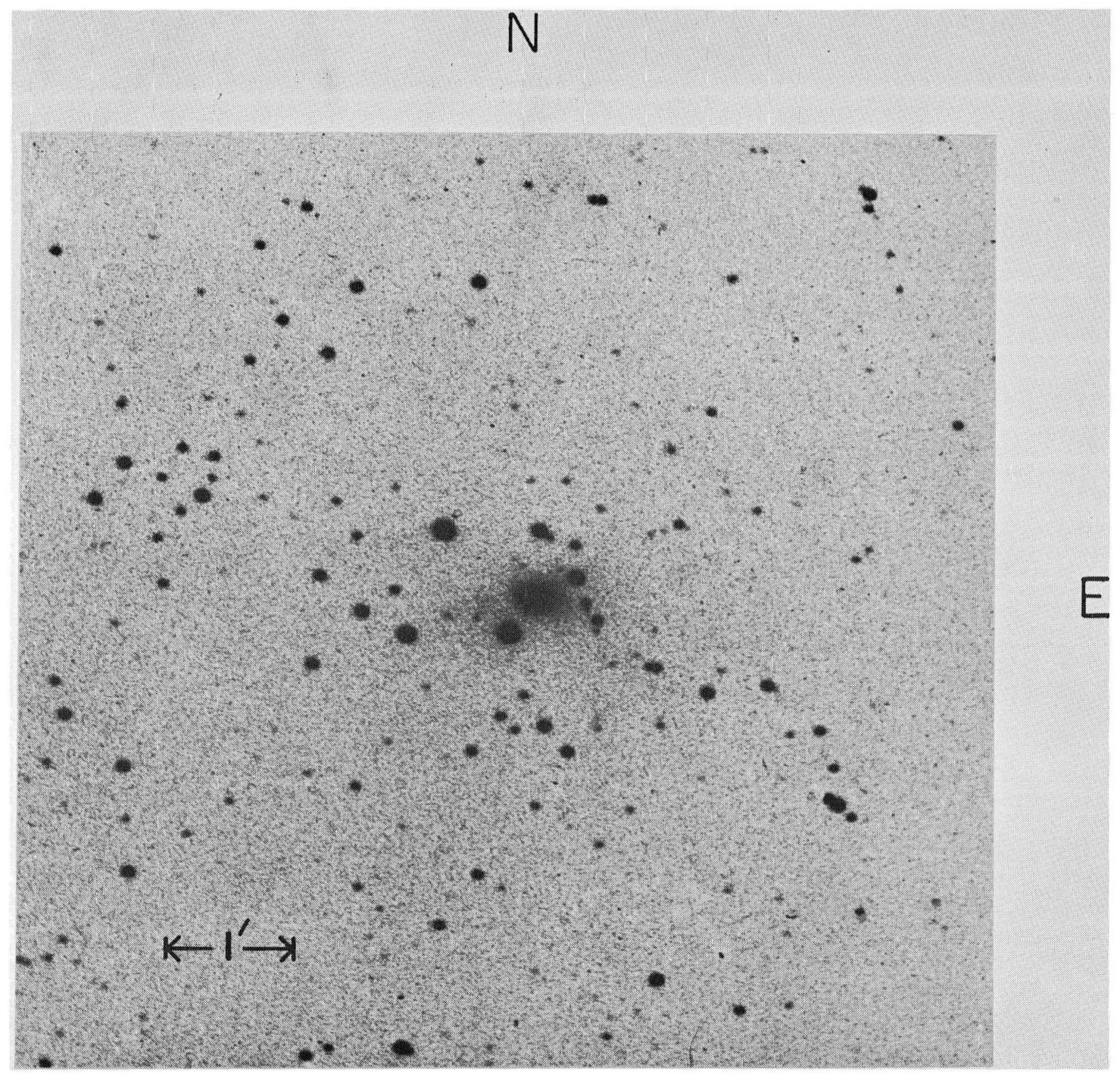

FIG. 1.-I-N plate of Maffei 1 obtained on the Leuschner 30-inch telescope (original scale $34^{\prime \prime} \mathrm{mm}^{-1}$ ), exposure 3 hours through an RG-8 filter.

SPINRAD et al. (see page L26) 


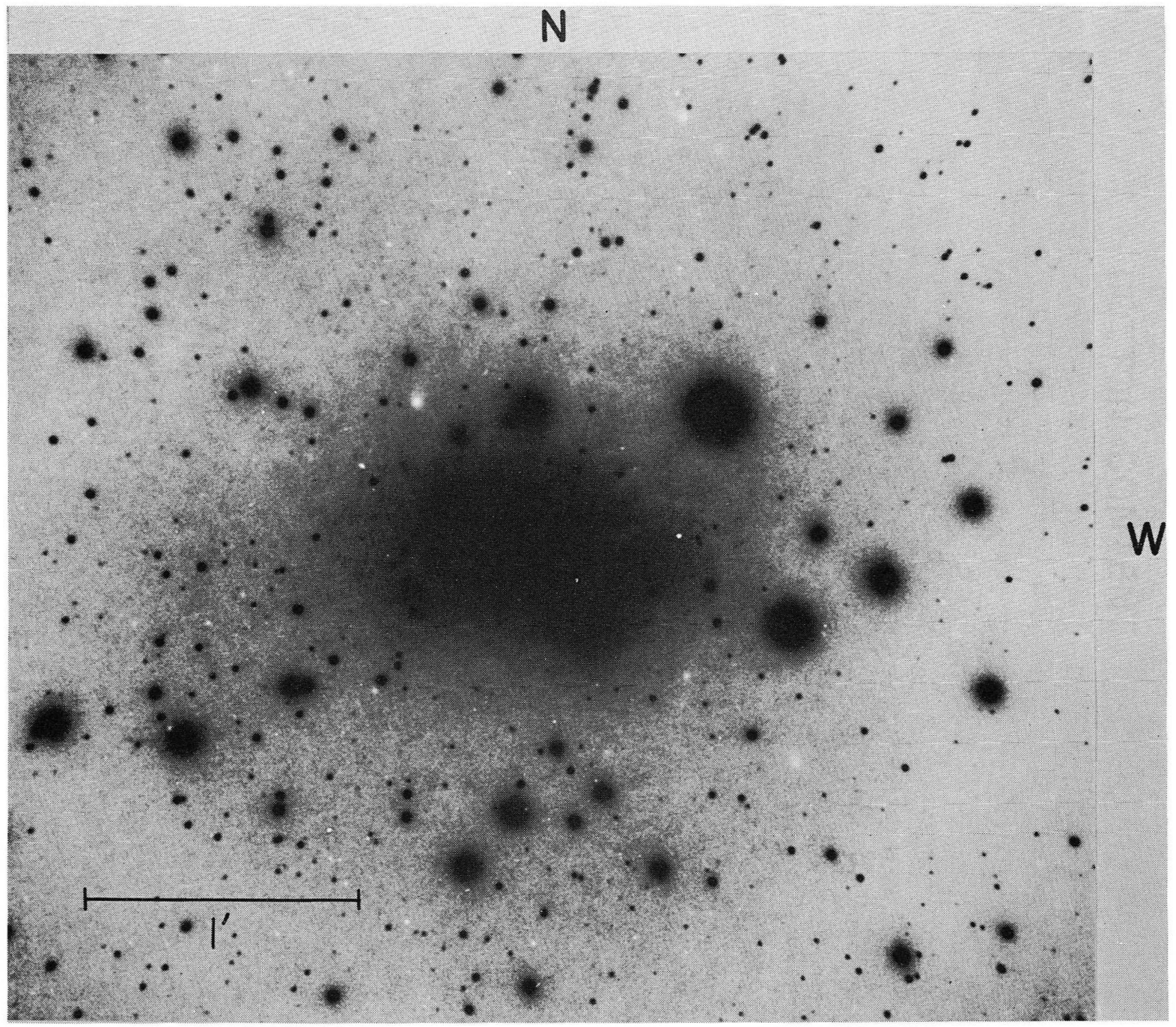

FIG. 2.-200-inch IIIa-J plate of Maffei 1 obtained with an ITT 4708 image intensifier and a Wratten 88A filter on the 200-inch telescope. The exposure time was 15 minutes. The resolution is better than $1^{\prime \prime}$.

SPINRAD et al. (see page L26) 
alternates between the signal from the object and that from a neighboring area of the sky; as a result, a correction must be applied to account for the finite signal from Maffei 1 in the "sky" reference. The correction, which is included in the data of Figure 3, was made by an iterative procedure using data obtained with a $10^{\prime \prime}$ aperture scanned across the object in an east-west direction. Typically, the correction was less than 10 percent.

\section{c) Photoelectric Spectrophotometry}

Maffei 1 was observed with the multichannel spectrometer on the 200-inch telescope on 1970 January 13-14. The seeing was around $2^{\prime \prime}$; an entrance aperture of $10^{\prime \prime}$ was used to make the observations. The exit slots were $80 \AA$ in the blue and $160 \AA$ in the red and near-infrared. The observations were reduced to absolute fluxes using the recent calibration of Oke and Schild (1970). The accuracy of the results for $\lambda>5000 \AA$ is 1 percent. For $\lambda<5000 \AA$ the uncertainty gradually increases to $\sim 2$ percent at $\lambda=$ $3950 \AA$. For $\lambda<3950 \AA$ no meaningful measures were obtained.

The absolute fluxes were corrected for interstellar reddening by using the Whitford reddening law (Whitford 1948) in such a way that the resulting color was similar to that of a normal giant elliptical galaxy and the nucleus of M31. This required a total absorption of $5.1 \pm 0.2 \mathrm{mag}$ at $\lambda=5556 \AA$. The results are shown in Figure 4 . Also shown is the energy distribution of the nuclei of giant ellipticals and M31 as given by Oke and Sandage (1968). Their magnitudes have been corrected to flux per unit frequency interval and to the new calibration of Oke and Schild (1970). The results, after some averaging of points in the far-red, are shown in Figure 4.

The overall energy distribution of the central $10^{\prime \prime}$ core of Maffei 1 out to $3.5 \mu$ was obtained by combining infrared photometry with the data obtained from the multichannel spectrometer and is also shown in Figure 4. Curve 15 of van de Hulst (1949) was used to obtain the reddening correction in the infrared.

The single-channel scanner data (not shown) also exhibit a red but composite spectrum. Specifically, the sodium D-lines and $\mathrm{TiO}$ bands have the line strengths commonly found in the nuclei of giant $\mathrm{E}, \mathrm{Sa}$, or Sb galaxies, rather than the weak appearance of dwarf systems like M32 and NGC 205.

\section{d) Slit Spectra}

Slit spectra of Maffei 1 show the Mg triplet lines commonly found in the yellow-red region of galaxies; a reproduction of a $60 \AA \mathrm{mm}^{-1}$ spectrum is shown in Figure 5 (Plate

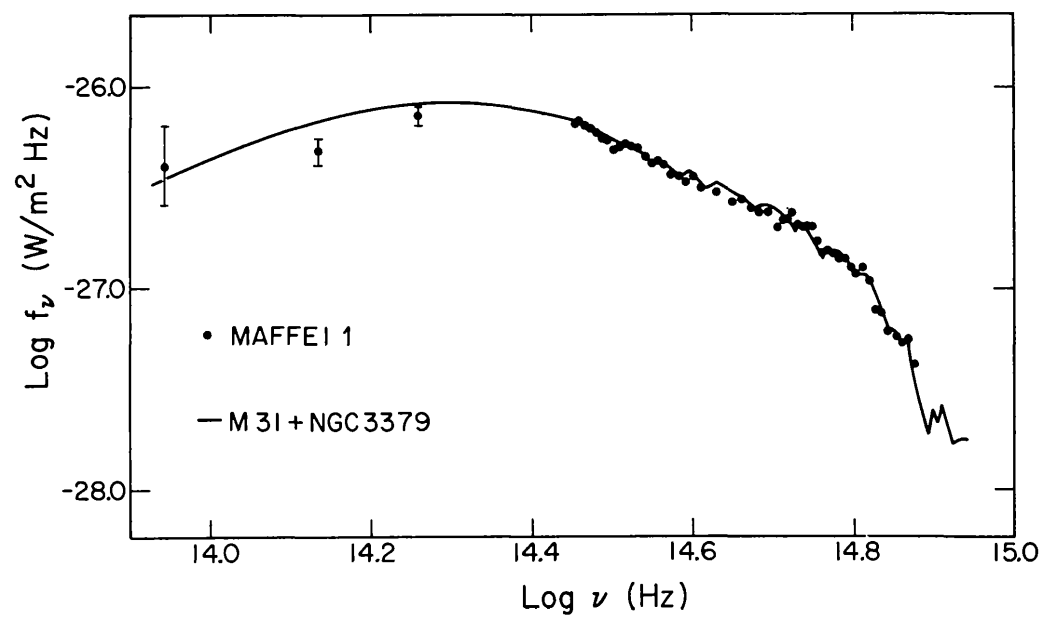

FIG. 4.-Energy distribution of Maffei 1, corrected for 5.2 magnitude of visual extinction, as determined by infrared and multichannel scanner data. Points, Maffei 1 data; solid line, the energy distribution for the nucleus of M31 and for NGC 3379. 


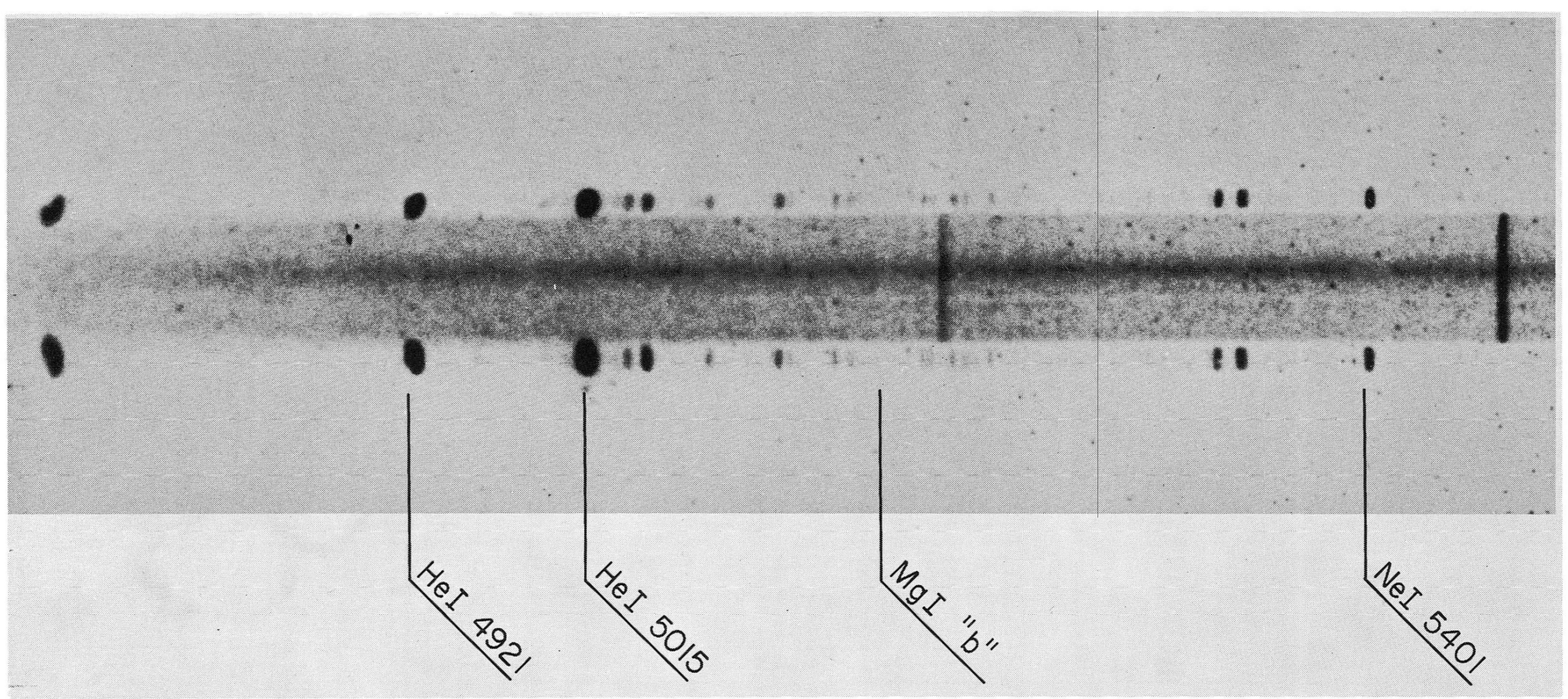

FIG. 5.- $60 \AA \mathrm{mm}^{-1}$ spectrogram of Maffei 1 obtained with the Cassegrain image-tube spectrograph of the 200-inch telescope. The Mg I $b$-lines are clearly visible. SPINRAD et al. (see page L27) 
L4). From the displacements of the lines in a core within $20^{\prime \prime}$ of the nucleus, a heliocentric velocity of $-10 \pm 50 \mathrm{~km} \mathrm{sec}^{-1}$ is obtained. The random velocity dispersion is estimated to be $200 \mathrm{~km} \mathrm{sec}^{-1}$; it is probably not less than $150 \mathrm{~km} \mathrm{sec}^{-1}$ or greater than $250 \mathrm{~km} \mathrm{sec}^{-1}$. The observed features also display a discontinuity across the nucleus, giving an estimated rotational velocity of $130 \mathrm{~km} \mathrm{sec}^{-1}$ averaged over the central $30^{\prime \prime}$ of the object.

\section{e) Radio Observations}

The 21-cm line of hydrogen was not detected (N. D., H. S.), to a limiting antenna temperature of about $0.1^{\circ} \mathrm{K}$, anywhere in the velocity ranges -500 to -175 and +50 to $+500 \mathrm{~km} \mathrm{sec}^{-1}$. Between -175 and $+50 \mathrm{~km} \mathrm{sec}^{-1}$ the galactic foreground makes it impossible to detect weak superimposed signals. The spread of internal velocities makes it extremely unlikely, however, that all the hydrogen in Maffei 1 could lie within this hidden range. If one accepts the optical rotational spread of $260 \mathrm{~km} \mathrm{sec}^{-1}$, then one end or the other of the velocity range would fall within the velocities at which the observations set an upper limit of $0.1^{\circ}$. At corresponding velocities M31 would give an antenna temperature of several degrees; hence we conclude that the mass of neutral hydrogen in Maffei 1 is much less than one-tenth that in M31.

\section{DISCUSSION}

\section{a) Identification}

The data presented above are strong circumstantial evidence that Maffei 1 is a large, normal elliptical galaxy; the evidence will be summarized below.

The photographs in Figure 1 show the form typical of an E3 or E4 galaxy. Densitometer tracings of the radial distribution of the surface brightness confirm that the rapid falloff from the center is more characteristic of elliptical galaxies such as NGC 4621 than of spirals but is, in fact, slower than in most ellipticals (see, e.g., Miller and Prendergast 1962). Both the large angular extent and the rapid drop in surface brightness with radial distance are confirmed by the infrared data of Figure 3.

The close similarity in detail between the energy distributions of Maffei 1 and M31 (Fig. 4) strongly suggests that Maffei 1 is a massive galaxy. Of particular interest is the question of whether the abrupt drop in flux for $\lambda<4030 \AA$, which is a striking feature of the nuclei of giant ellipticals M31, etc., is present in Maffei 1. This feature is produced largely by the $\mathrm{H}$ - and $\mathrm{K}$-lines of $\mathrm{Ca}$ II in late-type stars. Using the absolute fluxes for M31 for $\lambda>3950 \AA$ and the reddening already derived, one can predict the fluxes expected from Maffei 1 in this spectral range. From these one finds that if the true energy distribution were like that of M31, no signals would have been detected. The observations show this to be the case. Analysis of the actual photon counts in this spectral range shows that if the intrinsic fluxes from Maffei 1 had been significantly larger than those from M31, they would have been detected. Therefore, it can be concluded that in Maffei 1 there is a drop in flux for $\lambda<4030 \AA$ which is as large as or larger than that observed in M31.

The assumption that Maffei 1 is extragalactic requires that it be highly reddened, presumably by the Galaxy. The measurements obtained with the multichannel scanner require a visual absorption $A_{v}$ of $5.2 \pm 0.2 \mathrm{mag}$ in order for the continuum spectrum of Maffei 1 to duplicate that typical of the nuclei of $\mathrm{E}, \mathrm{S} 0$, and Sa galaxies. The singlechannel scanner data, taken by themselves, also yield a value $A_{v} \approx 5.2 \pm 0.2 \mathrm{mag}$ whereas the 2.2 - and $1.6-\mu$ photometry gives a value $A_{v} \sim 4.0 \pm 1.7$ mag. We adopt $A_{v}=5.2$ for subsequent discussion. This number is consistent with the normally adopted value of 1-2 mag visual absorption per kiloparsec within the Galaxy and the galactic coordinates of Maffei 1.

The absence of neutral-hydrogen radiation from Maffei 1, along with the large total mass found below, sets an upper limit to its fractional hydrogen mass and argues for its being an E, S0, or Sa galaxy. 


\section{b) Luminosity}

The integrated magnitude of Maffei 1 is obviously of interest; but it cannot be determined directly, because galactic obscuration hides all but the bright central regions. What one can do is to assume that the unobserved outer part of its profile resembles those of other galaxies which one can fit to the central profile of Maffei 1. For NGC 4621 (E4) the integrated brightness, as given by de Vaucouleurs (1965), is $134 I_{0} \theta_{c}{ }^{2}$, where $\theta_{c}$ is the core radius in seconds of arc, as defined above, and $I_{0}$ is the central surface brightness expressed as the number of twentieth-magnitude $(V)$ stars per square second. For NGC 4459 (S0), the proportionality factor is 76. Adopting a mean factor of 100 and the value for $\theta_{c}$ already found, and an (uncorrected) value for $I_{0}$ of 19.9 mag (arc sec) $)^{-2}$ from the scanner data, we get an integrated magnitude of $V=11.0$ for Maffei 1. With an absorption correction of $5.2 \mathrm{mag}$, this becomes $V_{0}=5.8$.

The infrared photometry as shown in Figure 3 apparently indicates a core radius smaller than 5 .5, although the data are not of sufficient accuracy to exclude the fit made to the photographic photometry. If a 5".5-radius core is assumed together with the energy distribution of Figure 3 , the data result in $V_{0}=6.5$; if the data are fitted by assuming a core radius of $2^{\prime \prime}$, then $V_{0}=7.7$. We provisionally adopt $V_{0}=5.8$ for subsequent discussion.

\section{c) Distance}

There are several methods of estimating the distance to Maffei 1; unfortunately, none is very reliable. An upper limit of $4 \mathrm{Mpc}$ can be placed on the distance if the intrinsic luminosity is not to exceed the $M_{V} \sim-22.5 \mathrm{mag}$ limit associated with giant $\mathrm{E}$ galaxies in clusters. Likewise, if the object were closer than $0.3 \mathrm{Mpc}$, its magnitude would be that of a dwarf galaxy; the lines in the spectrum are, however, stronger than for any known $\mathrm{dE}$ galaxy.

No estimate of high precision can be obtained from the observed galactocentric redshift. If one assumes a galactic circular velocity for the Sun of $250 \mathrm{~km} \mathrm{sec}^{-1}$, the radial velocity of Maffei 1 referred to the center of mass of the Galaxy is $165 \pm 50$ $\mathrm{km} \mathrm{sec}^{-1}$. If one now blindly applies the Hubble relation with $H=75 \mathrm{~km} \mathrm{sec}^{-1} \mathrm{Mpc}^{-1}$, the result of $2.2 \mathrm{Mpc}$ is achieved. If Maffei 1 is at this distance, the intrinsic visual magnitude would be $M_{V} \approx-20.5$, a not unreasonable value.

The most reliable distance estimate that can be made at present comes from dynamics. The method is in itself capable of fair accuracy, but the present data are of insufficient quality to give good results.

The gist of the method can be understood quite simply. From the virial theorem, the kinetic energy is proportional to the gravitational energy, or

$$
\frac{G \mathfrak{M}}{r} \propto\left\langle v^{2}\right\rangle
$$

where $v$ is the velocity dispersion in a pressure-supported system or the rotation velocity in a rotation-supported system, properly averaged, and $r$ is the radius of the system. The total mass $\mathfrak{M}$ is estimated from the luminosity by assuming a mass-to-light ratio $a$. The luminosity in turn is proportional to $R^{2} F$, where $F$ is the observed flux and $R$ the distance, so

$$
\frac{a G R^{2} F}{r} \propto\left\langle v^{2}\right\rangle \text {. }
$$

The system radius $r$ is $\theta R$, where $\theta$ is the appropriate angular radius, so finally

$$
R \propto \frac{\left\langle v^{2}\right\rangle \theta}{a G F} .
$$


The mass-to-light ratio adopted is $30 \mathfrak{M}_{\odot} / L_{\odot}$, consistent with that of giant elliptical galaxies and the nuclei of early-type spirals. Two approaches have been used in the application of the technique. The first uses the dynamics of the core region alone, and is insensitive to the exact behavior of the outer regions. It is clear that one can write equation (1) as

$$
R=C \frac{\left\langle v_{R}^{2}\right\rangle}{I_{0} \theta_{c} a}
$$

with $\theta_{c}$ and $I_{0}$ as previously defined, and $\left\langle v_{R}{ }^{2}\right\rangle$ the square of the observed radial-velocity dispersion. Theory, the details of which will be presented later (King and Minkowski 1971 ), gives $C=0.19\left[0.3+0.7\left(1-e^{2}\right)^{1 / 2}\right]^{-1}$, with $R$ in Mpc, $\theta_{c}$ in arc seconds, $\left\langle v_{R}^{2}\right\rangle^{1 / 2}$ in $\mathrm{km} \mathrm{sec}^{-1}$, and $a$ in solar units $(V)$. It is found that if $\theta_{c}$ is measured at $45^{\circ}$ to the projected major axis and $e$ is taken to be the projected eccentricity, the result is nearly independent of the true shape and orientation of the system. The result, with the parameters measured, is $R=0.9 \mathrm{Mpc}$.

In the second approach, use is made of the fact that the photographic and infrared photometry indicates that the isophotes of the object are roughly elliptical with nearly constant eccentricity 0.7 , and that the surface brightness falls off very nearly as $\theta^{-1}$ in any direction from the boundary of the core to a minute of arc or more. This allows a simple hydrodynamical model of the system to be constructed, which is a rotating generalization of the singular unbounded self-similar isothermal gas sphere. The model yields, for the observed eccentricity (and on the assumption that the observer is near the equatorial plane),

$$
R=\frac{1.0 \theta\left\langle v_{\phi}\right\rangle^{2}}{a G F_{\theta}}=\frac{0.5 \theta\left\langle v_{R}^{2}\right\rangle}{a G F_{\theta}}
$$

in cgs units, where $F_{\theta}$ is the flux in any circular diaphragm of radius $\theta>\theta_{c}$. Here $\left\langle v_{R}{ }^{2}\right\rangle$ is the velocity dispersion for a line of sight through the nucleus and $\left\langle v_{\phi}\right\rangle$ is the observed rotation velocity, which the model predicts is independent of $\theta$. The result from the rotation is $1.1 \mathrm{Mpc}$, and from the velocity dispersion $1.3 \mathrm{Mpc}$.

Considering the uncertainties and the fact that the methods refer to distinct portions of the system, the agreement is quite satisfactory. We adopt $1 \mathrm{Mpc}$ as a reasonable estimate of the distance. A meaningful error estimate is difficult to assign, since the dynamical data are of very poor quality, and $a$ is certainly not known to high precision. It seems rather unlikely that it could be wrong by more than a factor 2 ; perhaps it is reassuring that the estimate lies near the middle of the range established earlier by other considerations.

$$
\text { d) Mass }
$$

For a mass-luminosity ratio $a=30 \mathfrak{M}_{\odot} / L_{\odot}$, a distance of $1.2 \mathrm{Mpc}$, and $V_{0}$ of 5.8 , the mass of Maffei 1 is $2 \times 10^{11} \mathfrak{M}_{\odot}$, comparable to the mass of the Milky Way. It is clear from the way in which the distance and the mass are obtained, however, that the derived mass is proportional to $K\left\langle v_{r}^{2}\right\rangle^{2} / a I_{0}$, where $K$ is the proportionality constant between $I_{0} \theta_{c}{ }^{2}$ and the total brightness. The derived mass is also proportional to the correction made for interstellar absorption. All of these factors are uncertain-especially the fourth power of the velocity dispersion.

\section{e) Relationship to the Local Group}

If the estimates for the distance to Maffei 1 and for its mass are correct, Maffei 1 must be included in any consideration of the dynamics of the Local Group. In particular, with a galactocentric velocity of $165 \mathrm{~km} \mathrm{sec}^{-1}$ and a mass comparable to that of the Galaxy, Maffei 1 has a kinetic energy that exceeds that of any other known member.

Herbst (1969) has calculated the stability of the Local Group, not including Maffei 1. For the physically most reasonable case in which M31 and its satellites and the Galaxy 
and its satellites are considered bound subsystems, Herbst finds that the Local Group is marginally unstable and calculates $2 T /|\Omega|$ to be about $1.1 ; T$ is the total kinetic energy and $\Omega$ is the total potential energy of the Local Group. The Local Group is virially unstable if $2 T /|\Omega|$ is greater than unity.

Similar calculations have been made which include Maffei 1 as a member of the Local Group and which have used various values of the radial velocity, the mass, and the distance of Maffei 1. For a heliocentric radial velocity of $-10 \mathrm{~km} \mathrm{sec}^{-1}$, the smallest value of $2 T /|\Omega|$ consistent with the uncertainties in our determinations of mass and distance is 10, and therefore unless the Local Group contains appreciable mass in the form of intergalactic gas, as suggested by Oort (1970), Maffei 1 and the rest of the Local Group do not form a stable system. Even if the radial velocity were taken as low as -100 $\mathrm{km} \mathrm{sec}^{-1}, 2 T /|\Omega|$ is greater than 4 for all reasonable values of mass and distance.

It seems questionable to apply such notions as virial stability to the Local Group, however, since the characteristic dynamical times are at least as long as the Hubble time, perhaps longer. In particular, it is clear from simple geometric considerations that the "instability" simply implies that Maffei 1 is not bound to the Local Group; the stability of the rest of the system is not materially affected by its inclusion.

\section{CONCLUSIONS}

We conclude that Maffei 1 is a nearby elliptical galaxy, probably on the edge of the Local Group; its mass could be comparable to that of the Galaxy and M31. Its large kinetic energy apparently precludes its being bound to the Local Group.

More precise distance indicators are badly needed; in particular, future measures of sizes of any resolved objects-like globular clusters-in the infrared will be very useful. Observations of both Maffei 1 and Maffei 2 are being continued.

We would like to thank Rudolph Minkowski for advice on the determination of the velocity dispersion, A. R. Sandage and A. Toomre for valuable discussions, Malcolm Raff for providing the Leuschner plate used in our photometry, and Gary Tuton, Henry Tye, Harvey Butcher, and Ian Glass for their assistance.

This research was supported in part by National Science Foundation grants GP 14588 and GP 14356, and National Aeronautics and Space Administration grants NGL05-002-007 and NGL-05-002-134.

\section{REFERENCES}

Becklin, E. E., and Neugebauer, G. 1968, Ap. J., 151, 145.

Bell, M. B., Seaquist, E. R., and Braun, L. D. 1970, Ap. J. (Letters), 161, L13.

Herbst, W. 1969, Pub. A.S.P., 81, 619.

Hulst, H. C. van de. 1949, Rech. Astr. Obs. Utrecht, 11, Pt. 2.

Hyland, H., Becklin, E., Neugebauer, G., and Wallerstein, B. 1959, A p. J., 158, 619.

King, I. R., Byrnes, D., and Minkowski, R. 1967, A.J., 72, 306.

King, I. R., and Minkowski, R. 1971, in preparation.

Maffei, P. 1968, Pub. A.S.P., 80, 618.

Miller, R. H., and Prendergast, K. H. 1962, Ap. J., 136, 713.

Oke, J. B. 1969, Pub. A.S.P., 81, 11.

Oke, J. B., and Sandage, A. R. 1968, Ap. J., 154, 21.

Oke, J. B., and Schild, R. E. 1970,A p. J., 161, 1015.

Oort, J. H. 1970, Astr. and A P., 7, 381.

Vaucouleurs, G. de, and Vaucouleurs, A. de. 1965, Reference Catalogue of Bright Galaxies (Austin: University of Texas).

Wampler, E. J. 1968, Ap. J., 153, 19.

Whitford, A. E. 1948, Ap. J., 107, 102. 